\title{
MRI-Based Demonstration of The Normal Glymphatic System in a Human Population: A Systematic Review
}

Min Kyoung Lee

The Catholic University of Korea

Se Jin Cho

Seoul National University Bundang Hospital

Yun Jung Bae ( $\sim$ bae729@gmail.com )

Seoul National University Bundang Hospital

Jong-Min Kim

Seoul National University Bundang Hospital, Seoul National University College of Medicine

\section{Research Article}

Keywords: glymphatic system, CSF space, MRI, EMBASE databases, MEDLINE

Posted Date: June 17th, 2021

DOI: https://doi.org/10.21203/rs.3.rs-604443/v1

License: () (1) This work is licensed under a Creative Commons Attribution 4.0 International License. Read Full License 


\section{Abstract}

The glymphatic system has been described as the exchange between cerebrospinal fluid and interstitial fluid. Recently, many studies have demonstrated the presence of the glymphatic system based on MRI. The purpose of our study is to systematically review the studies demonstrating normal glymphatic system in a human population using MRI, and to find a detailed imaging protocol for the glymphatic system. We searched the MEDLINE and EMBASE databases to identify studies with human participants that contained MRI-based demonstrations of the normal glymphatic system. We extracted data on the imaging sequence, imaging protocol, and the targeted anatomical structures on each study. According to studies using contrast-enhanced MRI, peak enhancement was sequentially detected first in the CSF space, followed by the brain parenchyma, the meningeal lymphatic vessel (MLV), and finally the cervical lymph nodes, which corresponds to the glymphatic flow. Non-contrast flow-sensitive MRI showed similar results of glymphatic inflow from the CSF space to the brain parenchyma and efflux from the brain parenchyma to the MLV. Based on current systematic review, we recommended T1-weighted MRI using contrast material to visualize the glymphatic system. Our result can increase understanding of the glymphatic system and may lay the groundwork for future development of a standard imaging protocol.

\section{Introduction}

The lymphatic system plays a role in tissue homeostasis, interstitial fluid (ISF) clearance, and immune control ${ }^{1}$. Historically, the CSF has been thought to lack the connection to the lymphatic system ${ }^{1}$. However, proven interstitial solute clearance within the CNS and the presence of immune cells from the brain cannot be explained under the assumption of lacking lymphatic system, which has brought many studies investigating the possibility of a lymphatic-like system in the CNS ${ }^{1}$. As a result, Illiff et al. have firstly identified lymphatic system in the CNS for exchanging CSF and ISF via perivascular water flux, which is now called "glymphatic system" ${ }^{2-4}$. Many following studies have suggested that it could not only clear interstitial solute from CNS, but also induce extracellular fluid homeostasis and support the immune system within the CNS ${ }^{1,5,6}$.

Until now, most studies of the glymphatic system were animal studies performed using fluorescent tracers ${ }^{3,4,7}$. The animal-based studies $3,4,6,7$ have found that the glymphatic clearance flow starts within the CSF space and then is transported into the brain parenchyma. Here, fluid exchange between CSF and ISF occurs, and the exchanged fluid flows to the meningeal lymphatic vessel (MLV), and ultimately eliminated from CNS to the cervical lymph nodes (LN) (Supplementary Fig. 1). Although radiotracer studies for human population are limited, many recent studies have endeavored to reveal the presence of the glymphatic system in human and to visualize the glymphatic flow using diverse MRI-based techniques ${ }^{8-18}$. Indeed, several studies have demonstrated the glymphatic system in a human population, and even found out that dysfunction of the glymphatic system can be underlying pathophysiologic mechanism for natural brain aging and various CNS diseases ${ }^{2,19-21}$. But there is still a lack of systemic review for the glymphatic studies. This present study is the first systematic review of MRI-based publication to demonstrate the normal glymphatic system in human participants. We aimed to provide review on the imaging sequence, imaging protocol including contrast-agent injection protocol and acquisition time-points on dynamic contrast-enhanced scanning, and the targeted anatomical structures for measuring glymphatic system on each study.

\section{Materials And Methods}

This systematic review was performed according to the Preferred Reporting Items for Systematic reviews and Meta-Analyses (PRISMA) guidelines ${ }^{37}$.

Literature search

A systematic literature search of international databases MEDLINE, EMBASE, and COCHRANE Library was performed to identify studies of glymphatic demonstration in a normal population. Search terms were ([glymphatic] OR [meningeal lymphatic] OR [CNS lymphatics] OR [lymphatics of CNS] OR [cranial lymphatic]) AND (["magnetic resonance imaging"] OR ["MR imaging"] OR [MRI]). Our search was included studies that were published in English through April 13, 2020. To expand the search, the bibliographies of relevant articles were screened to identify other appropriate articles.

\section{Inclusion criteria}

Studies were included if they (1) involved study participants who are healthy volunteers for performing brain image or have no CNS disease other than CSF disorder or endolymphatic hydrops; (2) utilized imaging technique of MRl; and (3) contained results demonstrating the glymphatic system.

\section{Exclusion criteria}

Studies or subsets of studies were eliminated if they (1) were case reports with sample size less or equal to 10 patients; (2) were editorials, letters, abstracts, systematic reviews/meta-analyses, consensus statements, guidelines, or review articles; (3) included animal studies of the glymphatic system; (4) did not focus on demonstration of the glymphatic system; (5) were articles with (or suspected of having) overlapping populations; or (6) involved study participants with degenerative cerebral disease, hydrocephalus, traumatic brain injury, cerebrovascular disease, cerebral demyelinating disease, cerebral metabolic disease, epilepsy, and/or solid brain tumor.

\section{Data extraction}

We extracted the following data using standardized forms according to the PRISMA guidelines ${ }^{37}$ : (1) Study characteristics of author, year of publication, institution, country of origin, enrollment period, study design (prospective vs. retrospective), number of participants, mean participant age, male-to-female ratio, inclusion and exclusion criteria, and rationale of inclusion for each study; (2) MRI sequence used for glymphatic assessment: contrast-enhanced MRI using contrast-agent injection vs. non-contrast imaging sequence without necessity of contrast-agent injection (flow-sensitive MRI), type of contrast material and concentration, the method of contrast injection in contrast-enhanced MRI (intrathecal vs. intravenous), acquisition time-points for dynamic contrast-enhanced 
MRI, and MRI scanning parameters (MR machine vendor, magnetic field strength [Tesla], pulse sequences, repetition time, echo time, matrix size, field-of-view, slice thickness, and scan time); (3) Analytic method used in the glymphatic assessment: method of assessment (subjective visual assessment vs. objective signal quantification), targeted anatomical structures for the measurement, and glymphatic measurement targets (time-point dynamic enhancement curve and peak enhancement time in the contrast-enhanced MRI vs. flow change and direction in the flow-sensitive MRI). Particularly for the targeted anatomical structures for the glymphatic flow, we could include the four structures of CSF space, brain parenchyma, MLV, and cervical LN. We defined the CSF space as the subarachnoid space near the brain parenchyma. The ISF within the brain parenchyma was defined as the brain parenchyma in our research. The perivenous efflux drained to the MLV, and then the fluid finally moved the cervical LN (Supplementary Figure A). Therefore, we included MLV and cervical LN within the targeted anatomical structures. Two radiologists (X.X.X. and Y.Y.Y.) with seven years of experience in brain imaging independently searched the literature and selected appropriate studies. The discrepancy in two readers was solved by consensus.

\section{Quality assessment}

Two reviewers independently extracted the data and performed a quality assessment using the risk of bias for nonrandomized studies (RoBANS) tool for nonrandomized controlled trials ${ }^{38}$.

\section{Results}

Literature search

The study selection process is described in Fig. 1. We identified 1029 studies in our initial systematic search of the MEDLINE and EMBASE databases. No relevant trial was identified in the COCHRANE Library. After removing 258 duplicates, screening of the remaining 771 titles and abstracts yielded 20 potentially eligible articles. No additional articles were identified within the bibliographies of these articles. After full-text reviews of the 20 provisionally eligible articles, nine were excluded for the following reasons: two did not focus on demonstration of the glymphatic system ${ }^{22,23}$, two were reviews of articles 24,25 , two were abstracts ${ }^{26,27}$, and three included overlapping populations or those with a suspicion of overlapping ${ }^{28-30}$. Finally, 11 studies were included in our qualitative systematic review ${ }^{8-18}$.

\section{Characteristics of the included studies}

Table 1 shows the clinical characteristics of the 11 included studies. The total number of participants in these studies was 282 , with individual studies ranging from 5-190 participants. The age of each study ranged from 14-81 years. Seven studies included more women than men $8,10-12,14,17,18$, whereas two studies had more men than women ${ }^{13,15}$; the remaining two studies did not report sex distribution ${ }^{9,16}$. Seven studies had a prospective design $8,10-13,17,18$, and the other four studies were retrospective ${ }^{9,14-16}$. Among the selected studies, five contained healthy participants; therefore, we included all study participants from these studies 8,9,12,13,16. Meanwhile, six studies investigated both healthy participants and patients with neurological disorders; among patients with neurologic disorders, we only included patients who underwent an intrathecal contrast-enhanced MRI study for clinically suspicious spontaneous CSF leakage and who underwent a 4-hour-delayed intravenous contrast-enhanced MRI study for evaluation of endolymphatic hydrops also without other CNS pathology $10,11,14,15,17,18$. The detailed exclusion criteria for each of the studies are described in the footnote of Table 1. 
Table 1

The clinical characteristics of the included studies

\begin{tabular}{|c|c|c|c|c|c|c|c|c|c|}
\hline Group & Source & Affiliation & $\begin{array}{l}\text { Enrollment } \\
\text { period }\end{array}$ & $\begin{array}{l}\text { Study } \\
\text { Design }\end{array}$ & $\begin{array}{l}\text { Participant } \\
\text { no. }\end{array}$ & $\begin{array}{l}\text { Mean } \\
\text { age } \pm \text { SD } \\
\text { (range) }\end{array}$ & $\begin{array}{l}\text { Male: } \\
\text { Female }\end{array}$ & Inclusion/ Exclusion criteria & $\begin{array}{l}\text { Rationale } \\
\text { of Inclusion }\end{array}$ \\
\hline \multirow{8}{*}{$\begin{array}{l}\text { Contrast- } \\
\text { enhanced } \\
\text { MRI } \\
\text { group }\end{array}$} & $\begin{array}{l}\text { Absinta M } \\
\text { et al. } 2017\end{array}$ & $\begin{array}{l}\text { National } \\
\text { Institutes of } \\
\text { Health, USA }\end{array}$ & NA & Pros. & 5 & $\begin{array}{l}\text { NA }(28- \\
53)\end{array}$ & $2: 3$ & $\begin{array}{l}\text { In). Healthy participants/ Ex). } \\
\text { NA }\end{array}$ & $\begin{array}{l}\text { Healthy } \\
\text { participants }\end{array}$ \\
\hline & $\begin{array}{l}\text { Croci M et } \\
\text { al. } 2019\end{array}$ & $\begin{array}{l}\text { University of } \\
\text { Oxford, UK }\end{array}$ & NA & Retro. & 8 & NA & NA & $\begin{array}{l}\text { In). Healthy participantst/ Ex). } \\
\text { NA }\end{array}$ & $\begin{array}{l}\text { Healthy } \\
\text { participants }\end{array}$ \\
\hline & $\begin{array}{l}\text { Eide PK et } \\
\text { al. } 2018\end{array}$ & $\begin{array}{l}\text { Oslo } \\
\text { University } \\
\text { Hospital- } \\
\text { Rikshospitalet, } \\
\text { Norway }\end{array}$ & NA & Pros. & 16 & $\begin{array}{l}36.1 \pm \\
11.7 \\
(19-54)\end{array}$ & $2: 14$ & $\begin{array}{l}\text { In). CSF disordert (various) } \\
\text { with a deep cervical lymph } \\
\text { node with size }>1.5 \mathrm{~cm} / \mathrm{Ex} \text { ). } \\
\mathrm{H} / \mathrm{O} \text { hypersensitive reactions } \\
\text { to contrast media, } \mathrm{H} / \mathrm{O} \text { severe } \\
\text { allergy reactions, renal } \\
\text { dysfunction, pregnant or } \\
\text { breastfeeding women, and } \\
\text { age }<18 \text { YO or }>80 \text { YO }\end{array}$ & $\begin{array}{l}\text { To enroll } \\
\text { participants } \\
\text { with MRI } \\
\text { after i.t } \\
\text { contrast } \\
\text { injection, to } \\
\text { measure } \\
\text { the signal } \\
\text { change in } \\
\text { the cervical } \\
\text { LN }\end{array}$ \\
\hline & $\begin{array}{l}\text { Jacobsen } \\
\text { HH et al } \\
2019\end{array}$ & $\begin{array}{l}\text { Oslo } \\
\text { University } \\
\text { Hospital, } \\
\text { Norway }\end{array}$ & $\begin{array}{l}\text { February } \\
2016- \\
\text { August } \\
2018\end{array}$ & Pros. & 10 & $\begin{array}{l}36.9 \pm \\
6.95 \\
\text { (NA) }\end{array}$ & $2: 8$ & $\begin{array}{l}\text { In). CSF disorder (SIH or pineal } \\
\text { cyst)/ Ex). H/O } \\
\text { hypersensitivity reactions to } \\
\text { contrast media, } \mathrm{H} / 0 \text { severe } \\
\text { allergy reactions, renal } \\
\text { dysfunction, pregnant or } \\
\text { breastfeeding women, and } \\
\text { age }<18 \mathrm{YO} \text { or }>80 \mathrm{YO}\end{array}$ & $\begin{array}{l}\text { To enroll } \\
\text { participants } \\
\text { with MRI } \\
\text { after i.t } \\
\text { contrast } \\
\text { injection, to } \\
\text { measure } \\
\text { the signal } \\
\text { change } \\
\text { along the } \\
\text { visual tract }\end{array}$ \\
\hline & $\begin{array}{l}\text { Naganawa } \\
\text { S et al. } \\
2020\end{array}$ & $\begin{array}{l}\text { Nagoya } \\
\text { University } \\
\text { Graduate } \\
\text { School of } \\
\text { Medicine, } \\
\text { Japan }\end{array}$ & NA & Retro. & 190 & $\begin{array}{l}\text { NA }(14- \\
81)\end{array}$ & $91: 99$ & $\begin{array}{l}\text { In). Endolymphatic hydrops, } \\
\text { with } 4 \text { hours delayed MRI after } \\
\text { an i.v. injection of gadolinium/ } \\
\text { Ex). Brain tumor, cerebral } \\
\text { infarctions, } \mathrm{H} / \mathrm{O} \text { CNS infection, } \\
\text { and } \mathrm{H} / \mathrm{O} \text { recent systematic use } \\
\text { of steroid }\end{array}$ & $\begin{array}{l}\text { To enroll } \\
\text { participants } \\
\text { with } 4 \\
\text { hours delay } \\
\text { MRI after } \\
\text { i.v. contrast } \\
\text { injection } \\
\text { covering } \\
\text { the brain } \\
\text { and neck }\end{array}$ \\
\hline & $\begin{array}{l}\text { Oner AY et } \\
\text { al. } 2017\end{array}$ & $\begin{array}{l}\text { Gazi } \\
\text { University } \\
\text { School of } \\
\text { Medicine, } \\
\text { Turkey }\end{array}$ & $\begin{array}{l}1998- \\
2014\end{array}$ & Retro. & 6 & $\begin{array}{l}39.2 \pm \\
20.8 \\
(15-74)\end{array}$ & $6: 0$ & $\begin{array}{l}\text { In). CSF disorder (CSF } \\
\text { rhinorrhea or arachnoid cyst) } \\
\text { from cohorts of intrathecal CE- } \\
\text { MRC/ Ex). renal dysfunction, } \\
\text { transplantation, diabetes, and } \\
\text { malignancies }\end{array}$ & $\begin{array}{l}\text { To enroll } \\
\text { participants } \\
\text { with MRI } \\
\text { after i.t } \\
\text { contrast } \\
\text { injection }\end{array}$ \\
\hline & $\begin{array}{l}\text { Ringstad } \\
\text { G et al. } \\
2017\end{array}$ & $\begin{array}{l}\text { Oslo } \\
\text { University } \\
\text { Hospital- } \\
\text { Rikshospitalet, } \\
\text { Norway }\end{array}$ & $\begin{array}{l}\text { October } \\
\text { 2015-May } \\
2016\end{array}$ & Pros. & 8 & $\begin{array}{l}41.1 \pm \\
13.0 \\
\text { (NA) }\end{array}$ & $2: 6$ & $\begin{array}{l}\text { In). CSF disordert (CSF } \\
\text { leakage syndrome or } \\
\text { intracranial cyst)/ Ex). H/O } \\
\text { hypersensitive reactions to } \\
\text { contrast media, H/O severe } \\
\text { allergy reactions, renal } \\
\text { dysfunction, pregnant or } \\
\text { breastfeeding women, and } \\
\text { age }<18 \text { YO or }>80 \text { YO }\end{array}$ & $\begin{array}{l}\text { To enroll } \\
\text { participants } \\
\text { with MRI } \\
\text { after i.t } \\
\text { contrast } \\
\text { injection }\end{array}$ \\
\hline & $\begin{array}{l}\text { Zhou Y et } \\
\text { al. } 2020\end{array}$ & $\begin{array}{l}\text { Second } \\
\text { Affiliated } \\
\text { Hospital of } \\
\text { Zhejiang } \\
\text { University, } \\
\text { China }\end{array}$ & $\begin{array}{l}\text { April 2018- } \\
\text { November } \\
2019\end{array}$ & Pros. & 14 & $\begin{array}{l}55.9 \pm \\
12.7(20- \\
71)\end{array}$ & $6: 8$ & $\begin{array}{l}\text { In). CSF disorder (leakage) or } \\
\text { peripheral neuropathy from } \\
\text { cohorts of lumbar puncture } \\
\text { and voluntary participationt/ } \\
\text { Ex). H/O hypersensitivity } \\
\text { reactions to contrast media, } \\
\text { H/O severe allergy reactions, } \\
\text { renal dysfunction, and } \\
\text { pregnant or breastfeeding } \\
\text { women }\end{array}$ & $\begin{array}{l}\text { To enroll } \\
\text { participants } \\
\text { with MRI } \\
\text { after i.t } \\
\text { contrast } \\
\text { injection }\end{array}$ \\
\hline
\end{tabular}

Abbreviations:

CE-MRC, contrast-enhanced MR cisternography; CNS, central nervous system; CSF, cerebrospinal fluid; Ex)., exclusion; H/O, history of; In)., inclusion; i.t, intrathecal; i.v.; intravenous; LN, lymph node; MMSE, mini-mental state examination; MRI, magnetic resonance image; NA, not applicable; Pros., Prospective; SD, standard deviation; SIH, spontaneous intracranial hypotension; Retro., Retrospective; YO, Years old

Footnotes: * According to the MRI sequence for analysis; +We excluded following participants: Eide PK et al., three patients with hydrocephalus; Croci M et al., fifteen patients with hydrocephalus; Ringstad $\mathrm{G}$ et al., fifteen patients with hydrocephalus; Zhou Y et al., twenty-three patients with brain diseases, including cerebrovascular disease, neurodegenerative disease, hydrocephalus, encephalitis, and metabolic disease; Rajna Z et al., ten patients with Alzheimer disease 


\begin{tabular}{|c|c|c|c|c|c|c|c|c|c|}
\hline Group & Source & Affiliation & $\begin{array}{l}\text { Enrollment } \\
\text { period }\end{array}$ & $\begin{array}{l}\text { Study } \\
\text { Design }\end{array}$ & $\begin{array}{l}\text { Participant } \\
\text { no. }\end{array}$ & $\begin{array}{l}\text { Mean } \\
\text { age } \pm \text { SD } \\
\text { (range) }\end{array}$ & $\begin{array}{l}\text { Male: } \\
\text { Female }\end{array}$ & Inclusion/ Exclusion criteria & $\begin{array}{l}\text { Rationale } \\
\text { of Inclusion }\end{array}$ \\
\hline \multirow[t]{3}{*}{$\begin{array}{l}\text { Non- } \\
\text { contrast } \\
\text { MRI } \\
\text { group }\end{array}$} & $\begin{array}{l}\text { Kiviniemi } \\
\text { V et al. } \\
2016\end{array}$ & $\begin{array}{l}\text { Oulu } \\
\text { University } \\
\text { Hospital, } \\
\text { Finland }\end{array}$ & NA & Pros. & 9 & $\begin{array}{l}25.67 \pm \\
4.52 \\
(\mathrm{NA})\end{array}$ & $4: 5$ & $\begin{array}{l}\text { In). Healthy participants/ Ex). } \\
\text { NA }\end{array}$ & $\begin{array}{l}\text { Healthy } \\
\text { participants }\end{array}$ \\
\hline & $\begin{array}{l}\text { Kuo PH et } \\
\text { al. } 2018\end{array}$ & $\begin{array}{l}\text { University of } \\
\text { Arizona, USA }\end{array}$ & NA & Pros. & 6 & $\begin{array}{l}\text { NA }(30- \\
56)\end{array}$ & $4: 2$ & $\begin{array}{l}\text { In). Healthy participants/ Ex). } \\
\text { NA }\end{array}$ & $\begin{array}{l}\text { Healthy } \\
\text { participants }\end{array}$ \\
\hline & $\begin{array}{l}\text { Rajna Z et } \\
\text { al. } 2019\end{array}$ & $\begin{array}{l}\text { Oulu } \\
\text { University } \\
\text { Hospital, } \\
\text { Finland }\end{array}$ & NA & Retro. & 10 & $\begin{array}{l}58.3 \pm \\
9.9(\mathrm{NA})\end{array}$ & NA & $\begin{array}{l}\text { In). Healthy participantst/ Ex). } \\
\text { NA }\end{array}$ & $\begin{array}{l}\text { Healthy } \\
\text { participants }\end{array}$ \\
\hline \multicolumn{10}{|c|}{ Abbreviations: } \\
\hline \multicolumn{10}{|c|}{$\begin{array}{l}\text { CE-MRC, contrast-enhanced MR cisternography; CNS, central nervous system; CSF, cerebrospinal fluid; Ex)., exclusion; H/O, history of; In)., inclusion; i.t, } \\
\text { intrathecal; i.V.; intravenous; LN, lymph node; MMSE, mini-mental state examination; MRI, magnetic resonance image; NA, not applicable; Pros., Prospective; } \\
\text { SD, standard deviation; SIH, spontaneous intracranial hypotension; Retro., Retrospective; YO, Years old }\end{array}$} \\
\hline \multicolumn{10}{|c|}{$\begin{array}{l}\text { Footnotes: * According to the MRI sequence for analysis; +We excluded following participants: Eide PK et al., three patients with hydrocephalus; Croci M et } \\
\text { al., fifteen patients with hydrocephalus; Ringstad } \mathrm{G} \text { et al., fifteen patients with hydrocephalus; Zhou Y et al., twenty-three patients with brain diseases, } \\
\text { including cerebrovascular disease, neurodegenerative disease, hydrocephalus, encephalitis, and metabolic disease; Rajna Z et al., ten patients with } \\
\text { Alzheimer disease }\end{array}$} \\
\hline
\end{tabular}

\section{MRI sequence for glymphatic assessment}

Table 2 shows the detailed MRI sequence and protocol for glymphatic assessment. The studies were categorized into two groups-a contrast-enhanced MRI group and a flow-sensitive MRI group-according to the use of contrast injection. The contrast-enhanced MRI group was defined as those who were performed post-contrast MRI acquisition after intrathecal or intravenous contrast-agent injection. The non-contrast MRI group was defined as those who did not undergo contrast-agent injection by any route, but was performed flow-sensitive MRI including time-of-flight MR angiograph (TOF-MRA) and phase-contrast MRI. The contrast-enhanced MRI group ( 8 studies) assessed the presence of the glymphatic system by detecting contrast-enhancement within the glymphatic system $8-11,14,15,17,18$, while the non-contrast MRI group (3 studies) evaluated the glymphatic system using delineation of the CSF flow (through the glymphatic system) ${ }^{12,13,16}$. Among the eight studies in the contrast-enhanced MRI group, seven used intrathecal contrast injection ${ }^{8-11,15,17,18}$, while only one administered contrast agent as an intravenous injection ${ }^{14}$. Most contrast agents were gadolinium-based extracellular fluid agents, including gadobutrol $8,10,11,14,17$, gadopentetate dimeglumine ${ }^{15}$, and gadodiamide ${ }^{18}$, except one study that did not clarify the type of contrast agent ${ }^{9}$. Five of the 8 studies in the contrast-enhanced MRI group performed time-point dynamic observations within the targeted structures in the glymphatic system ${ }^{9-11,17,18}$, whereas the other three studies in the contrast group ${ }^{8,14,15}$ and all studies in the non-contrast MRI group ${ }^{12,13,16}$ did not perform time-point dynamic observations. The details regarding the time schedules of the time-point dynamic observations are shown in Table 2. 
Table 2

MRI sequence used for glymphatic assessment

\begin{tabular}{|c|c|c|c|c|c|c|c|c|c|c|c|}
\hline \multirow[t]{2}{*}{ Group } & \multirow[t]{2}{*}{ Source } & \multirow{2}{*}{$\begin{array}{l}\text { Contrast } \\
\text { Material } \\
\text { (concentrate) }\end{array}$} & \multirow{2}{*}{$\begin{array}{l}\text { Method } \\
\text { of } \\
\text { Contrast } \\
\text { Injection }\end{array}$} & \multirow{2}{*}{$\begin{array}{l}\text { Dynamic } \\
\text { observation }\end{array}$} & \multirow{2}{*}{$\begin{array}{l}\text { Time } \\
\text { schedule in } \\
\text { dynamic } \\
\text { observation }\end{array}$} & \multicolumn{6}{|c|}{ Detailed MR protocol } \\
\hline & & & & & & $\begin{array}{l}\text { Vendor, } \\
\text { Machine }\end{array}$ & $\mathbf{T}$ & MR sequence & TR/TE & Matrix & $\begin{array}{l}\text { FO' } \\
\text { (mI }\end{array}$ \\
\hline \multirow[t]{8}{*}{$\begin{array}{l}\text { Contrast- } \\
\text { enhancement } \\
\text { MRI group }\end{array}$} & $\begin{array}{l}\text { Absinta M } \\
\text { et al. } 2017\end{array}$ & $\begin{array}{l}\text { Gadobultrol } \\
(0.1 \mathrm{mmol} / \mathrm{ml})\end{array}$ & i.t & No & NA & $\begin{array}{l}\text { Skyra, } \\
\text { Siemens }\end{array}$ & 3 & $\begin{array}{l}\text { T1WI (black } \\
\text { blood,SPACE); } \\
\text { FLAIR } \\
\text { (SPACE) }\end{array}$ & $\begin{array}{l}938 / 22 \\
4800 / 354\end{array}$ & $\begin{array}{l}512 \times \\
512 \\
320 x \\
320\end{array}$ & $\begin{array}{l}174 \\
174 \\
235 \\
235\end{array}$ \\
\hline & $\begin{array}{l}\text { Croci M et } \\
\text { al. } 2019\end{array}$ & NA & i.t & Yes & $\begin{array}{l}1,3,4.5 \\
\text { and } 24 \mathrm{~h}\end{array}$ & NA & NA & NA & NA & NA & NA \\
\hline & $\begin{array}{l}\text { Eide PK et } \\
\text { al. } 2018\end{array}$ & $\begin{array}{l}\text { Gadobultrol } \\
(1.0 \mathrm{mmol} / \mathrm{ml})\end{array}$ & i.t & Yes & $\begin{array}{l}\text { Pre., } 2-4, \\
4-6,6-9 \\
24, \text { and } 48 \mathrm{~h}\end{array}$ & $\begin{array}{l}\text { Ingenia, } \\
\text { Siemens }\end{array}$ & 3 & $\mathrm{~T} 1 \mathrm{WI}$ & $5.1 / 2.3$ & $\begin{array}{l}256 \times \\
256\end{array}$ & $\begin{array}{l}512 \\
512\end{array}$ \\
\hline & $\begin{array}{l}\text { Jacobsen } \\
\text { HH et al } \\
2019\end{array}$ & $\begin{array}{l}\text { Gadobutrol } \\
(1.0 \mathrm{mmol} / \mathrm{ml})\end{array}$ & i.t & Yes & $\begin{array}{l}0-20,20- \\
40, \text { and } 40- \\
60 \mathrm{~min}, 1- \\
2,2-4,4- \\
6,6-9,24 \\
\text { and } 48 \mathrm{~h}\end{array}$ & $\begin{array}{l}\text { Ingenia, } \\
\text { Philips }\end{array}$ & 3 & $\mathrm{~T} 1 \mathrm{WI}$ & $5.1 / 2.3$ & $\begin{array}{l}256 \times \\
256\end{array}$ & $\begin{array}{l}25 \epsilon \\
25 \epsilon\end{array}$ \\
\hline & $\begin{array}{l}\text { Naganawa } \\
\text { S et al. } \\
2020\end{array}$ & $\begin{array}{l}\text { Gadobutrol } \\
(0.1 \mathrm{mmol} / \mathrm{ml})\end{array}$ & i.v & No & $4 \mathrm{~h}$ & $\begin{array}{l}\text { Skyra, } \\
\text { Siemens }\end{array}$ & 3 & $\begin{array}{l}\text { FLAIR } \\
\text { (SPACE); }\end{array}$ & $15130 / 549$ & $\begin{array}{l}324 \times \\
384\end{array}$ & $\begin{array}{l}165 \\
19 \epsilon\end{array}$ \\
\hline & $\begin{array}{l}\text { Oner AY et } \\
\text { al. } 2017\end{array}$ & $\begin{array}{l}\text { Gadopentetate } \\
\text { dimeglumine } \\
\text { (NA) }\end{array}$ & i.t & No & NA & $\begin{array}{l}\text { Excite, } \\
\text { GE; } \\
\text { Verio, } \\
\text { Siemens }\end{array}$ & $\begin{array}{l}1.5 \\
3\end{array}$ & $\mathrm{~T} 1 \mathrm{WI}$ & $550 / 12$ & $\begin{array}{l}288 \times \\
160\end{array}$ & 20 \\
\hline & $\begin{array}{l}\text { Ringstad } \\
\text { G et al. } \\
2017\end{array}$ & $\begin{array}{l}\text { Gadobutrol } \\
(1.0 \mathrm{mmol} / \mathrm{ml})\end{array}$ & i.t & Yes & $\begin{array}{l}\text { Pre, } 0-20, \\
20-40 \text {, and } \\
40-60 \text { min, } \\
1-2,2-4 \\
4-6,6-9, \\
\text { and } 24 \mathrm{~h}\end{array}$ & $\begin{array}{l}\text { Ingenia, } \\
\text { Philips }\end{array}$ & 3 & T1WI & $5.1 / 2.3$ & $\begin{array}{l}256 \times \\
256\end{array}$ & $\begin{array}{l}25 \epsilon \\
25 \epsilon\end{array}$ \\
\hline & $\begin{array}{l}\text { Zhou Y et } \\
\text { al. } 2020\end{array}$ & $\begin{array}{l}\text { Gadodiamide } \\
(0.5 \mathrm{mmol} / \mathrm{ml})\end{array}$ & i.t & Yes & $\begin{array}{l}4.5,15 \text {, and } \\
39 \mathrm{~h}\end{array}$ & $\begin{array}{l}\text { GE 750, } \\
\text { GE }\end{array}$ & 3 & $\begin{array}{l}\text { FLAIR (2D } \\
\text { and 3D } \\
\text { CUBE)- }\end{array}$ & $\begin{array}{l}8400 / 152 \\
5000 / 131\end{array}$ & $\begin{array}{l}320 x \\
320 \\
256 x \\
256\end{array}$ & $\begin{array}{l}18 \\
18 \\
23 . \\
23 .\end{array}$ \\
\hline \multirow[t]{3}{*}{$\begin{array}{l}\text { Non-contrast } \\
\text { MRI group }\end{array}$} & $\begin{array}{l}\text { Kiviniemi } \\
\text { V et al. } \\
2016\end{array}$ & No & No & No & NA & $\begin{array}{l}\text { Skyra, } \\
\text { Siemens }\end{array}$ & 3 & MREG & $100 / 36$ & $\begin{array}{l}64 \times \\
64 \times \\
64\end{array}$ & NA \\
\hline & $\begin{array}{l}\text { Kuo PH et } \\
\text { al. } 2018\end{array}$ & No & No & No & NA & $\begin{array}{l}\text { Skyra, } \\
\text { Siemens }\end{array}$ & 3 & TOF MRA & $30 / 4.49$ & $\begin{array}{l}160 \times \\
160\end{array}$ & 50 \\
\hline & $\begin{array}{l}\text { Rajna Z et } \\
\text { al. } 2019\end{array}$ & No & No & No & NA & $\begin{array}{l}\text { Skyra, } \\
\text { Siemens }\end{array}$ & 3 & MREG & $100 / 1.4$ & NA & NA \\
\hline
\end{tabular}

Abbreviations: \& and; 2D, two-dimension; 3D, three-dimension; CE-MRC, contrast-enhanced MR cisternography; CE-T1WI, contrast-enhanced T1-weighted ima! resolution 3D turbo spin echo acquisition GE; DWI, diffusion weighted image; FLAIR, fluid attenuated inversion recovery; FOV, field-of-view; i.t, intrathecal; i.v., i encephalography(ultra-fast 3D k-space under-sampling technique); MPRAGE, magnetization prepared rapid gradient echo as a high resolution whole brain T1 applicable; ; ROI, region of interest; SPACE, sampling perfection with application-optimized contrasts by using different flip angle evaluation as a high resoluti acquisition in Siemens; SSS, superior sagittal sinus; ; T, tesla; T1WI, T1-weighed image; T2WI, T2-weighted image; TE, echo time; TOF MRA, Time-of-Flight MR time

Of the studies included in the contrast-enhanced MRI group, six performed MRI examinations using 3.0-T scanners ${ }^{8,10,11,14,17,18}$, one study carried out the MRI examinations using either 1.5- or 3.0-T scanner ${ }^{15}$, and one study did not mention the magnetic field strength ${ }^{9}$. All studies in the non-contrast MRI group performed MRI examinations using 3.0-T scanners ${ }^{12,13,16}$. All studies in the contrast-enhanced MRI group evaluated contrast enhancement on conventional images, including T1-weighted images or fluid attenuated inversion recovery (FLAIR) images ${ }^{8-11,14,15,17,18}$. However, the imaging parameters for each sequence differed between studies; the detailed MRI parameters are described in Table 2. Regarding three studies in the non-contrast MRI group, two utilized phase-contrast MR encephalography for CSF flow assessment ${ }^{12,13}$, while the third performed TOF-MRA for CSF flow demonstration (Table 2).

\section{Analytic method for glymphatic assessment}

Table 3 shows the analytic method used for glymphatic assessment. Seven of the included studies used a method of objective signal quantification by measuring the signal ratio change or flow change ${ }^{9-12,16-18}$, while three of the included studies used a subjective visual assessment of contrast enhancement or flow change ${ }^{8,13,14}$; the final study used both subjective and objective analyses ${ }^{15}$. Targeted anatomic structures for the glymphatic measurement are described in Table 3. Among the contrast-enhanced MRI group, five studies assessed contrast signal changes in multiple locations ${ }^{9-11,17,18}$; one study at all four locations ${ }^{18}$, one study at three locations (CSF space, brain parenchyma, and cervical LN) ${ }^{10}$, and three studies at two locations (CSF space and brain parenchyma) 9,11,17. The two studies in the non-contrast MRI group assessed the flow-signal change at multiple locations, including CSF space, brain 
parenchyma, and MLV ${ }^{12,16}$. The other four studies (comprising three contrast-enhanced MRI studies and one non-contrast MRI study) examined the signal change at only one location: the CSF space in one study ${ }^{14}$, the brain parenchyma in one study ${ }^{15}$, and the MLV in two studies ${ }^{8,13}$. The most common locations for signal measurement were the CSF space ${ }^{9-12,14,16-18}$ and the brain parenchyma ${ }^{9-12,15-18}$ in eight studies each. The MLV was evaluated in five studies $8,12,13,16,18$, and the cervical LN were assessed in two studies ${ }^{10,18}$. Time-point dynamic observations were performed in the contrast-enhanced MRI group but not in the non-contrast MRI group. All five studies that carried out time-point dynamic observations in the contrast-enhanced MRI group assessed the signal ratio change and detected peak enhancement time at multiple anatomic locations ${ }^{9-11,17,18}$. As a result, the peak enhancement times following contrast injection according to targeted structure were 4-6 hours within the CSF space (range: 3-9 hours), 24-48 hours within the brain parenchyma (range: 15-48 hours), 15 hours within the MLV (range: 15-39 hours), and 24-48 hours within the cervical LN (range: 24-48 hours). 
Table 3

Analytic method used in the glymphatic assessment of each sequence

\begin{tabular}{|c|c|c|c|c|c|c|c|c|}
\hline \multirow[t]{2}{*}{ Group } & \multirow[t]{2}{*}{ Source } & \multirow{2}{*}{$\begin{array}{l}\text { Subj. } \\
\text { vs. } \\
\text { Obj. }\end{array}$} & \multicolumn{4}{|c|}{ Targeted anatomical structures } & \multirow{2}{*}{$\begin{array}{l}\text { Time-point } \\
\text { dynamic } \\
\text { enhancement } \\
\text { and peak } \\
\text { enhancement } \\
\text { time }\end{array}$} & \multirow{2}{*}{$\begin{array}{l}\text { Flow change } \\
\text { and direction }\end{array}$} \\
\hline & & & CSF & Parenchyma & MLV & Cervical LN & & \\
\hline \multirow{6}{*}{$\begin{array}{l}\text { Contrast- } \\
\text { enhancement } \\
\text { MRI group }\end{array}$} & $\begin{array}{l}\text { Absinta M } \\
\text { et al. } 2017\end{array}$ & Subj. & No & No & Yes (5 of 5$)$ & No & NA & NA \\
\hline & $\begin{array}{l}\text { Croci M et } \\
\text { al. } 2019\end{array}$ & Obj. & $\begin{array}{l}\text { Yes } \\
\text { (foramen magnum } \\
(3 \mathrm{~h}) \text {, Sylvian fissure } \\
(5 \mathrm{~h}) \text {, and precentral } \\
\text { sulcus (7h)) }\end{array}$ & $\begin{array}{l}\text { Yes } \\
\text { (gray matter in IFG) }\end{array}$ & No & No & $\begin{array}{l}\text { Peak } \\
\text { glymphatic } \\
\text { enhancement } \\
\text { occurred } \\
\text { within all CSF } \\
\text { spaces and } \\
\text { brain } \\
\text { parenchyma } \\
\text { (gray matter in } \\
\text { IFG) after }<7 \mathrm{~h} \\
\text { and } 15 \mathrm{~h}^{\ddagger}\end{array}$ & NA \\
\hline & $\begin{array}{l}\text { Eide PK et } \\
\text { al. } 2018\end{array}$ & Obj. & $\begin{array}{l}\text { Yes } \\
\text { (near IFG after 4-9h } \\
\text { in } 13 \text { of } 16 \\
\text { individuals) }\end{array}$ & $\begin{array}{l}\text { Yes } \\
\left(\text { IFG (in } 14 \text { of } 15^{*}\right) \text {, } \\
\text { PHG, thalamus, and } \\
\text { pons after } 24-48 \mathrm{~h}\end{array}$ & No & $\begin{array}{l}\text { Yes } \\
\text { (LN after } \\
24-48 \mathrm{~h} \text { in } 9 \\
\text { of } 15^{\star} \\
\text { individuals) }\end{array}$ & $\begin{array}{l}\text { Peak } \\
\text { glymphatic } \\
\text { enhancement } \\
\text { predominantly } \\
\text { occurred } \\
\text { within CSF } \\
\text { space (near } \\
\text { IFG), brain } \\
\text { parenchyma } \\
\text { (IFG), and } \\
\text { cervical LN } \\
\text { T1Wl after 4- } \\
\text { 6h, 24-48h, } \\
\text { and 24-48h }\end{array}$ & NA \\
\hline & $\begin{array}{l}\text { Jacobsen } \\
\text { HH et al } \\
2019\end{array}$ & Obj. & $\begin{array}{l}\text { Yes } \\
\text { (prechiasmatic } \\
\text { cistern after 4-6h) }\end{array}$ & $\begin{array}{l}\text { Yes } \\
\text { (optic nerve, optic } \\
\text { chiasm, optic tract, } \\
\text { and primary visual } \\
\text { cortex after } 24 h \text {, } \\
\text { except optic chiasm } \\
(6-9 \mathrm{~h}) \text { ) }\end{array}$ & No & No & $\begin{array}{l}\text { Peak } \\
\text { glymphatic } \\
\text { enhancement } \\
\text { occurred } \\
\text { within CSF } \\
\text { (prechiasmatic } \\
\text { cistern) and } \\
\text { brain } \\
\text { parenchyma } \\
\text { of visual } \\
\text { pathway (optic } \\
\text { nerve, optic } \\
\text { tract, and } \\
\text { primary visual } \\
\text { cortex) on } \\
\text { T1WI after 4- } \\
\text { 6h and 24h }\end{array}$ & NA \\
\hline & $\begin{array}{l}\text { Naganawa } \\
\text { S et al. } \\
2020\end{array}$ & Subj & $\begin{array}{l}\text { Yes } \\
\text { (around the cortical } \\
\text { veins after } 4 \mathrm{~h} \text { in } \\
155 \text { of } 190 \\
\text { individuals) }\end{array}$ & No & No & No & $\begin{array}{l}\text { Glymphatic } \\
\text { enhancement } \\
\text { occurred } \\
\text { within CSF } \\
\text { space around } \\
\text { the cortical } \\
\text { vein on FLAIR } \\
\text { after } 4 \mathrm{~h}\end{array}$ & NA \\
\hline & $\begin{array}{l}\text { Oner AY et } \\
\text { al. } 2017\end{array}$ & $\begin{array}{l}\text { Subj. } \\
\& \\
\text { Obj. }\end{array}$ & No & $\begin{array}{l}\text { Yes } \\
\text { (globus pallidus and } \\
\text { dentate nucleus in } 5 \\
\text { of } 6 \\
\text { individuals/Increment } \\
\text { of signal intensity } \\
\text { ratio within brain } \\
\text { parenchyma on T1WI } \\
(6 \text { of } 6)+\end{array}$ & No & No & $\begin{array}{l}\text { Glymphatic } \\
\text { enhancement } \\
\text { occurred } \\
\text { within brain } \\
\text { parenchyma } \\
\text { (dentate } \\
\text { nucleus and } \\
\text { globus } \\
\text { pallidus) on } \\
\text { T1Wl }\end{array}$ & NA \\
\hline
\end{tabular}

Abbreviations: \&, and; ACA, anterior cerebral artery; h, hours; IFG, inferior frontal gyrus; LN, lymph node; MCA, middle cerebral artery; min, minutes; MLV, meningeal lymphatic vessels; NA, not appliable; PCA, posterior cerebral artery; PHG, parahippocampal gyrus; pre., precontrast; Obj., objective signal quantification; SSS, superior sagittal sinus; sub., subjective visual assessment; TOF MRA, time of flight MR angiography

Footnotes: * The number of parent patients' were not same to original enrolled number of patients due the peak enhancement can only evaluated in the person who showed positive signal units change:18 (not 19) in parenchyma 17 (not 19) in cervical LN in Eide PK et al. 2018, and 2 (not 14) in cervical LN in Zhou Y et al. 2020; † The increment of globus pallidus-to-thalamus signal intensity ratio and dentate nucleus-to-pons signal intensity ratio was evaluated between initial unenhanced T1WI and control unenhanced T1WI after the performance of CE-MRC; ¥The white matter did not reach peak enhancement within $24 \mathrm{~h}$. 


\begin{tabular}{|c|c|c|c|c|c|c|c|c|}
\hline \multirow[t]{2}{*}{ Group } & \multirow[t]{2}{*}{ Source } & \multirow{2}{*}{$\begin{array}{l}\text { Subj. } \\
\text { vs. } \\
\text { Obj. }\end{array}$} & \multicolumn{4}{|c|}{ Targeted anatomical structures } & \multirow{2}{*}{$\begin{array}{l}\text { Time-point } \\
\text { dynamic } \\
\text { enhancement } \\
\text { and peak } \\
\text { enhancement } \\
\text { time }\end{array}$} & \multirow{2}{*}{$\begin{array}{l}\text { Flow change } \\
\text { and direction }\end{array}$} \\
\hline & & & CSF & Parenchyma & MLV & Cervical LN & & \\
\hline & $\begin{array}{l}\text { Ringstad } \\
\text { G et al. } \\
2017\end{array}$ & Obj. & $\begin{array}{l}\text { Yes } \\
\text { (foramen magnum } \\
(1-2 \mathrm{~h}), \text { pontine } \\
\text { cistern (1-2h), } \\
\text { Sylvian fissure (4- } \\
6 \mathrm{~h}), 3 \mathrm{rd} \text { and 4th } \\
\text { ventricles (4-6h), } \\
\text { central sulcus (4- } \\
6 \mathrm{~h}), \text { and lateral } \\
\text { ventricle (6-9h), and } \\
\text { after }<9 \mathrm{~h} \text { ) }\end{array}$ & $\begin{array}{l}\text { Yes } \\
\text { (IFG, pons, thalamus, } \\
\text { frontal horn, and } \\
\text { precentral gyrus after } \\
24 \mathrm{~h} \text { ) }\end{array}$ & No & No & $\begin{array}{l}\text { Peak } \\
\text { glymphatic } \\
\text { enhancement } \\
\text { occurred } \\
\text { within all CSF } \\
\text { spaces and } \\
\text { brain } \\
\text { parenchyma } \\
\text { (IFG) on T1WI } \\
\text { after }<9 \mathrm{~h} \text { and } \\
24 \mathrm{~h}\end{array}$ & NA \\
\hline & $\begin{array}{l}\text { Zhou Y et } \\
\text { al. } 2020\end{array}$ & Obj. & $\begin{array}{l}\text { Yes } \\
\text { (4th (in } 10 \text { of 14), } \\
3^{\text {rd }} \text { (in } 9 \text { of 14), and } \\
\text { lateral ventricles (in } \\
9 \text { of 14) after } 4.5 \mathrm{~h} \text { ) }\end{array}$ & $\begin{array}{l}\text { Yes } \\
\text { (frontal horn (in } 10 \text { of } \\
14 \text { ), IFG (in } 11 \text { of } 14 \text { ), } \\
\text { and precentral gyrus } \\
\text { (in } 12 \text { of } 14 \text { ) after } 15 \\
\text { h) }\end{array}$ & $\begin{array}{l}\text { Yes } \\
\text { (after } 15 \mathrm{~h} \text { in } \\
9 \text { of } 14 \\
\text { individuals) }\end{array}$ & $\begin{array}{l}\text { Yes } \\
\text { (cervical LN } \\
\text { after } 39 \mathrm{~h} \text { in } \\
2 \text { of } 2^{*} \\
\text { individuals) }\end{array}$ & $\begin{array}{l}\text { Peak } \\
\text { glymphatic } \\
\text { enhancement } \\
\text { occurred } \\
\text { within CSF } \\
\text { space (fourth } \\
\text { ventricle), } \\
\text { brain } \\
\text { parenchyma } \\
\text { (precentral } \\
\text { gyrus), MLV } \\
\text { on FLAIR, and } \\
\text { cervical LN on } \\
\text { T1Wl after } \\
\text { 4.5h, 15h, 15h, } \\
\text { and 39h }\end{array}$ & NA \\
\hline \multirow[t]{2}{*}{$\begin{array}{l}\text { Non-contrast } \\
\text { MRI group }\end{array}$} & $\begin{array}{l}\text { Kiviniemi } \\
\text { V et al. } \\
2016\end{array}$ & Obj. & $\begin{array}{l}\text { Yes } \\
\text { (periarterial) }\end{array}$ & Yes & $\begin{array}{l}\text { Yes } \\
\text { (perivenous) }\end{array}$ & No & NA & $\begin{array}{l}\text { Glymphatic } \\
\text { flows were } \\
\text { demonstrated } \\
\text { within brain } \\
\text { parenchyma on } \\
\text { MREG (arterial } \\
\text { pulsation } \\
\text { induced } \\
\text { glymphatic flow } \\
\text { from CSF } \\
\text { spaces to the } \\
\text { brain } \\
\text { parenchyma } \\
\text { and venous } \\
\text { flow induce } \\
\text { glymphatic flow } \\
\text { from the brain } \\
\text { parenchyma to } \\
\text { MLV) }\end{array}$ \\
\hline & $\begin{array}{l}\text { Kuo PH et } \\
\text { al. } 2018\end{array}$ & Subj. & No & No & $\begin{array}{l}\text { Yes } \\
\text { (alongside } \\
\text { the SSS in } 6 \\
\text { of } 6 \\
\text { individuals) }\end{array}$ & No & NA & $\begin{array}{l}\text { Glymphatic } \\
\text { flows were } \\
\text { demonstrated } \\
\text { within MLV on } \\
\text { TOF MRA (MLV } \\
\text { had } \\
\text { countercurrent } \\
\text { flow to venous } \\
\text { flow) }\end{array}$ \\
\hline
\end{tabular}

Abbreviations: \&, and; ACA, anterior cerebral artery; h, hours; IFG, inferior frontal gyrus; LN, lymph node; MCA, middle cerebral artery; min, minutes; MLV, meningeal lymphatic vessels; NA, not appliable; PCA, posterior cerebral artery; PHG, parahippocampal gyrus; pre., precontrast; Obj., objective signal quantification; SSS, superior sagittal sinus; sub., subjective visual assessment; TOF MRA, time of flight MR angiography

Footnotes: * The number of parent patients' were not same to original enrolled number of patients due the peak enhancement can only evaluated in the person who showed positive signal units change:18 (not 19) in parenchyma 17 (not 19) in cervical LN in Eide PK et al. 2018, and 2 (not 14) in cervical LN in Zhou Y et al. 2020; † The increment of globus pallidus-to-thalamus signal intensity ratio and dentate nucleus-to-pons signal intensity ratio was evaluated between initial unenhanced T1WI and control unenhanced T1WI after the performance of CE-MRC; $\neq$ The white matter did not reach peak enhancement within $24 \mathrm{~h}$. 


\begin{tabular}{|c|c|c|c|c|c|c|c|c|}
\hline \multirow{2}{*}{\multicolumn{2}{|c|}{ Group }} & \multirow{2}{*}{$\begin{array}{l}\text { Subj. } \\
\text { vs. } \\
\text { Obj. }\end{array}$} & \multicolumn{4}{|c|}{ Targeted anatomical structures } & \multirow{2}{*}{$\begin{array}{l}\text { Time-point } \\
\text { dynamic } \\
\text { enhancement } \\
\text { and peak } \\
\text { enhancement } \\
\text { time }\end{array}$} & \multirow{2}{*}{$\begin{array}{l}\text { Flow change } \\
\text { and direction }\end{array}$} \\
\hline & & & CSF & Parenchyma & MLV & Cervical LN & & \\
\hline & $\begin{array}{l}\text { Rajna Z et } \\
\text { al. } 2019\end{array}$ & Obj. & Yes & Yes & Yes & No & NA & $\begin{array}{l}\text { Glymphatic } \\
\text { flows were } \\
\text { demonstrated } \\
\text { within brain } \\
\text { parenchyma on } \\
\text { MREG (arterial } \\
\text { pulsation } \\
\text { induced } \\
\text { glymphatic flow } \\
\text { from CSF } \\
\text { spaces to the } \\
\text { brain } \\
\text { parenchyma) }\end{array}$ \\
\hline \multicolumn{9}{|c|}{$\begin{array}{l}\text { Abbreviations: \&, and; ACA, anterior cerebral artery; h, hours; IFG, inferior frontal gyrus; LN, lymph node; MCA, middle cerebral artery; min, minutes; MLV, } \\
\text { meningeal lymphatic vessels; NA, not appliable; PCA, posterior cerebral artery; PHG, parahippocampal gyrus; pre., precontrast; Obj., objective signal } \\
\text { quantification; SSS, superior sagittal sinus; sub., subjective visual assessment; TOF MRA, time of flight MR angiography }\end{array}$} \\
\hline \multicolumn{9}{|c|}{$\begin{array}{l}\text { Footnotes: }{ }^{*} \text { The number of parent patients' were not same to original enrolled number of patients due the peak enhancement can only evaluated in the } \\
\text { person who showed positive signal units change: } 18 \text { (not } 19 \text { ) in parenchyma } 17 \text { (not } 19 \text { ) in cervical LN in Eide PK et al. } 2018 \text {, and } 2 \text { ( not } 14 \text { ) in cervical LN } \\
\text { in Zhou Y et al. } 2020 ; \dagger \text { The increment of globus pallidus-to-thalamus signal intensity ratio and dentate nucleus-to-pons signal intensity ratio was } \\
\text { evaluated between initial unenhanced T1WI and control unenhanced T1Wl after the performance of CE-MRC; } ¥ \text { The white matter did not reach peak } \\
\text { enhancement within } 24 \mathrm{~h} \text {. }\end{array}$} \\
\hline
\end{tabular}

In the non-contrast MRI group, two studies objectively measured flow change on MR encephalography ${ }^{12,16}$, and one study visualized flow change on TOFMRA ${ }^{15}$. Arterial pulsation induced centrifugal flow in the CSF space, while venous pulsation induced centripetal flow in the MLV. Moreover, the MLV had countercurrent flow to venous flow.

\section{Assessment of the study quality}

A quality assessment of the included studies was performed according to the RoBANS criteria and is presented in Fig. 2 . All 11 studies showed a low risk of bias in participant comparability, confounding variables, incomplete outcome data, and selective reporting. However, one study showed an unclear risk of bias in selection of participants, as they did not clearly explain the enrollment period of their retrospective study ${ }^{14}$. In measurement of exposure, two studies showed an unclear risk of bias because of the undescribed measurement methods ${ }^{8,9}$. Five of the 11 studies showed an unclear risk of bias in blinding of the outcome assessment due to unclear statements from the readers who underwent radiologic assessment $8,9,12,13,16$. Finally, in the outcome evaluation, two studies showed an unclear risk of bias because they did not contain a clear statement about their imaging analysis ${ }^{8,13}$.

\section{Discussion}

This systematic review showed the current research trends of the glymphatic system. The main concepts of the included studies were observation of contrastenhancement or CSF flow changes within one or multiple anatomical structures of the glymphatic system. Among the 11 included studies, eight were categorized into the contrast-enhanced MRI group, and three were placed in the non-contrast MRI group. For assessment of the glymphatic system, five studies from the contrast-enhanced MRI group were used to perform a time-point dynamic observation of contrast-enhancement within certain targeted locations and demonstrated peak enhancement time. In contrast, three studies from the non-contrast MRI group carried out TOF-MRA or phase-contrast MR encephalography to demonstrate CSF flow within the glymphatic system. Arterial flow induced glymphatic influx from the CSF space to the brain parenchyma, and venous flow induced glymphatic efflux from the brain parenchyma to the MLV.

The traditional concept of the CNS system holds that this system lacks an anatomically defined lymphatic system to assist in CSF-ISF exchange ${ }^{1}$. However, recent studies have suggested the presence of anatomical pathways between the CSF and ISF space, and this CSF-ISF exchange should play a role in clearing ISF solute ${ }^{4-6}$ from the CNS. In animal studies $4,7,31,32$, researchers have observed the glymphatic system using fluorescent CSF tracers and have shown that CSF tracers entered the brain parenchyma from the CSF space and were cleared through the MLV, then the CSF tracers drained into the deep cervical LN 4,7 .

On the other hand, to assess the glymphatic system in a human population, many studies have utilized various MRI techniques to observe glymphatic enhancement and/or glymphatic flow ${ }^{8-18}$. Especially, recent studies have demonstrated an association between dysfunction in the glymphatic system and variable neurologic disease including traumatic brain injury, Alzheimer's disease, and normal pressure hydrocephalus as well as the normal aging ${ }^{2,19-21}$. In particular, it has been suggested that the impairment of the glymphatic system may be a potential cause for developing normal pressure hydrocephalus and Alzheimer's disease $9,17,21$. In this regard, acknowledgement of the presence of the glymphatic system is expected to provide a potential therapeutic direction for many neurodegenerative diseases.

However, although the concept of a glymphatic system is a newly emerging and clinically meaningful topic, no systematic review for the MRI-based glymphatic system in a human population has been performed so far. Thus, through this systematic review, we aimed to verify the current state of research on the glymphatic system, to improve the robustness of the methodology, and to lay groundwork for future development of a standard imaging protocol for in vivo study. The main strength of this systematic review is that it is the first to demonstrate the glymphatic system in a human population without prior and present CNS pathology. 
To be specific the glymphatic system was described as the function of the ISF exchange in the CNS system, and many previous studies ${ }^{5-7}$ have reported that the glymphatic system begins in the CSF space and sequentially flows into the brain parenchyma, MLV, and cervical LN (Supplementary Fig. 1A and B).

Studies in the contrast-enhanced MRI group revealed peak enhancement time first in the CSF space, followed by the brain parenchyma and MLV, and finally in the cervical LN. This peak enhancement pattern was similar to those of previous studies of glymphatic system flow patterns revealed by the animal radiotracer examinations ${ }^{6,7}$, which can verify the demonstration of the glymphatic system presence on the MRI. Moreover, the results of the non-contrast MRI group revealed similar CSF flow patterns in the glymphatic system. They demonstrated that arterial pulsation induces centrifugal flow in the CSF space, while venous pulsation induces centripetal flow in the MLV. In addition, the MLV has countercurrent flow to venous flow. Similar to the change in peak enhancement time on contrast-enhanced MRI, flow change began in the CSF space and extended toward the brain parenchyma and MVL on non-contrast MRI. Therefore, these results can also support the presence of glymphatic pathway in a human population. This verification of the glymphatic flow on contrast-enhanced and non-contrast MRIs could have significant clinical value, because it can be applied to the detection of the glymphatic dysfunction which may reveal the underlying pathophysiology of several neuro-degenerative diseases including Alzheimer disease, normal pressure hydrocephalus, etc. Therefore, we believe that this systematic review could provide the basis for the future studies linking glymphatic function and cerebral degenerative disease.

Based on the relevant studies, we should give the method using contrast-agent superiority over the non-contrast flow-sensitive MRI for the glymphatic imaging, since assessing CSF flow change on non-contrast MRI is a subjective analysis, while contrast-enhanced MRI can not only provide subjective analysis but also objective measurement for the degree of the glymphatic flow changes. Therefore, we highly recommend contrast-enhanced T1-weighted MRI using gadolinium-based contrast-agent for the glymphatic dedicated imaging. With regard to the intrathecal injection of the contrast-agent, we recommend the following time schedule for measuring peak enhancement within the glymphatic system; CSF within $4 \sim 6$ hours, parenchyma and MLV within $15 \sim 48$ hours, and cervical lymph node more than 24 hours. However, as for the intravenous contrast-agent injection, we could not specifically recommend optimal target structure or time schedule, because there was only one study using intravenous contrast-agent injection. Considering that intravenous contrast-agent injection is much accessible than intrathecal injection in clinical practice, future studies using intravenous injection are warranted to widen its usage to the normal population, and thus to make standard glymphatic imaging protocol and the suitable time schedule for intravenous approach.

Our study had several limitations. First, we included only a small number of studies that investigated study participants without underlying CNS pathology. However, when considering the intrathecal contrast injection is seldomly performed on the selective patients with CSF leakage due to the risk of chemical meningitis and neurotoxicity ${ }^{33-36}$, it was inevitable to include a small number of subjects. Second, we included only one study that performed intravenous contrast injection to assess the glymphatic system. This study evaluated contrast enhancement observation at a single time and location. Therefore, it is difficult to draw conclusions about glymphatic flow timing and pattern in studies that used intravenous contrast injection. However, since intravenous contrast

injection is safer than intrathecal injection ${ }^{34,35}$, future large studies using intravenous injection will contribute to the ever-growing body of glymphatic research. Third, although we made effort to enroll studies that demonstrate glymphatic system within normal healthy population without having significant CNS disorder, studies that performed intrathecal injection included patients who were suspected to have CSF leakage. However, although these patients may not seem as normal healthy population technically, they did not have underlying CNS disease, and their CSF leakage was induced by minor trauma; therefore, we decided to include these patients in our systemic review. Similarly, we included patients with endolymphatic hydrops which is a peripheral vestibular disease, but those without any underlying CNS disease. Therefore, we believe that we could successfully enroll patients without CNS disorders close to normal healthy population. Therefore, we enrolled studies included patients with suspicious CSF leakage or endolymphatic hydrops.

The present systematic review showed imaging sequence, imaging protocol including contrast-agent injection protocol and acquisition time-points on dynamic contrast-enhanced scanning, and the targeted anatomical structures for measuring glymphatic system. Our findings could enhance the understanding of the human glymphatic system and the current MRI protocols for measuring glymphatic flow. Based on the review, we recommend contrastenhanced T1-weighted MRI over non-contrast flow-sensitive MRI for the glymphatic imaging We believe that future studies linking cerebral degenerative disease and glymphatic functioning can be built on the basis of our current study.

\section{Declarations}

\section{Acknowledgements: None}

Competing interests: The authors report no competing interests.

\section{Conflict of interest}

The authors of this manuscript declare no relationships with any companies, whose products or services may be related to the subject matter of the article.

Funding: No direct funding was received for the work covered in this study.

\section{Informed consent:}

Written informed consent was not required for this study because this study is systematic review.

\section{Ethical approval:}

Institutional Review Board approval was not required because this study is systematic review.

\section{Author contributions}




\begin{tabular}{|lll|}
\hline Name & Location & Contribution \\
\hline $\begin{array}{l}\text { Min } \\
\text { Kyoung }\end{array}$ & $\begin{array}{l}\text { Department of Radiology, Yeouido St. Mary's Hospital, College of } \\
\text { Medicine, The Catholic University of Korea }\end{array}$ & $\begin{array}{l}\text { Study concept and design, acquisition and interpretation of data, and } \\
\text { drafted the manuscript for intellectual content }\end{array}$ \\
\hline $\begin{array}{l}\text { Se Jin } \\
\text { Cho }\end{array}$ & $\begin{array}{l}\text { Department of Radiology, Seoul National University Bundang } \\
\text { Hospital }\end{array}$ & $\begin{array}{l}\text { Study concept and design, acquisition and interpretation of data, statistical } \\
\text { analysis, and revised the manuscript for intellectual content }\end{array}$ \\
\hline $\begin{array}{l}\text { Yun } \\
\text { Jung } \\
\text { Bae }\end{array}$ & $\begin{array}{l}\text { Department of Radiology, Seoul National University Bundang } \\
\text { Hospital }\end{array}$ & $\begin{array}{l}\text { Providing updated data, interpretation of data, and revised the manuscript } \\
\text { for intellectual content }\end{array}$ \\
\hline $\begin{array}{l}\text { Jong- } \\
\text { Min } \\
\text { Kim }\end{array}$ & $\begin{array}{l}\text { Department of Neurology, Seoul National University Bundang } \\
\text { Hospital, Seoul National University College of Medicine }\end{array}$ & Providing updated data and revised the manuscript for intellectual content \\
\hline
\end{tabular}

\section{References}

1. Trevaskis, N. L., Kaminskas, L. M. \& Porter, C. J. From sewer to saviour-targeting the lymphatic system to promote drug exposure and activity. Nature Reviews Drug Discovery 14, 781-803 (2015).

2. Iliff, J. J. et al. Impairment of glymphatic pathway function promotes tau pathology after traumatic brain injury. Journal of Neuroscience $\mathbf{3 4}$, $16180-16193$ (2014).

3. Iliff, J. J. et al. Brain-wide pathway for waste clearance captured by contrast-enhanced MRI. The Journal of clinical investigation 123, 1299-1309 (2013).

4. Iliff, J. J. et al. A paravascular pathway facilitates CSF flow through the brain parenchyma and the clearance of interstitial solutes, including amyloid B. Science translational medicine 4, 147ra111-147ra111 (2012).

5. Engelhardt, B. et al. Vascular, glial, and lymphatic immune gateways of the central nervous system. Acta neuropathologica 132, 317-338 (2016).

6. Plog, B. A. \& Nedergaard, M. The glymphatic system in central nervous system health and disease: past, present, and future. Annual Review of Pathology: Mechanisms of Disease 13, 379-394 (2018).

7. Aspelund, A. et al. A dural lymphatic vascular system that drains brain interstitial fluid and macromolecules. Journal of Experimental Medicine 212, 991 999 (2015).

8. Absinta, M. et al. Human and nonhuman primate meninges harbor lymphatic vessels that can be visualized noninvasively by MRI. Elife 6 (2017).

9. Croci, M., Vinje, V. \& Rognes, M. E. Uncertainty quantification of parenchymal tracer distribution using random diffusion and convective velocity fields. Fluids and Barriers of the CNS 16 (2019).

10. Eide, P. K., Vatnehol, S. A. S., Emblem, K. E. \& Ringstad, G. Magnetic resonance imaging provides evidence of glymphatic drainage from human brain to cervical lymph nodes. Scientific reports 8, 7194 (2018).

11. Jacobsen, H. H. et al. The human visual pathway communicates directly with the subarachnoid space. Investigative Ophthalmology and Visual Science 60, 2773-2780 (2019).

12. Kiviniemi, V. et al. Ultra-fast magnetic resonance encephalography of physiological brain activity - Glymphatic pulsation mechanisms? J Cereb Blood Flow Metab 36, 1033-1045 (2016).

13. Kuo, P. H., Stuehm, C., Squire, S. \& Johnson, K. Meningeal Lymphatic Vessel Flow Runs Countercurrent to Venous Flow in the Superior Sagittal Sinus of the Human Brain. Tomography 4, 99-104 (2018).

14. Naganawa, S. et al. Confirmation of Age-dependence in the Leakage of Contrast Medium around the Cortical Veins into Cerebrospinal Fluid after Intravenous Administration of Gadolinium-based Contrast Agent. Magn Reson Med Sci (2020).

15. Oner, A. Y., Barutcu, B., Aykol, S. \& Tali, E. T. Intrathecal Contrast-Enhanced Magnetic Resonance Imaging-Related Brain Signal Changes: Residual Gadolinium Deposition? Invest Radio/ 52, 195-197 (2017).

16. Rajna, Z. et al. 3D Multi-Resolution Optical Flow Analysis of Cardiovascular Pulse Propagation in Human Brain. IEEE Trans Med Imaging 38, 2028-2036 (2019).

17. Ringstad, G. \& Eide, P. K. Cerebrospinal fluid tracer efflux to parasagittal dura in humans. Nature Communications 11 (2020).

18. Zhou, Y. et al. Impairment of the Glymphatic Pathway and Putative Meningeal Lymphatic Vessels in the Aging Human. Ann Neuro/ 87, 357-369 (2020).

19. Kress, B. T. et al. Impairment of paravascular clearance pathways in the aging brain. Annals of neurology 76, 845-861 (2014).

20. Peng, W. et al. Suppression of glymphatic fluid transport in a mouse model of Alzheimer's disease. Neurobiology of disease $93,215-225$ (2016).

21. Ringstad, G., Vatnehol, S. A. S. \& Eide, P. K. Glymphatic MRI in idiopathic normal pressure hydrocephalus. Brain 140, 2691-2705 (2017).

22. Demiral Ş, B. et al. Apparent diffusion coefficient changes in human brain during sleep - Does it inform on the existence of a glymphatic system? Neurolmage 185, 263-273 (2019).

23. Yamada, S., Ishikawa, M., Yamamoto, K., Yamaguchi, M. \& Oshima, M. Location-specific characteristics of perivascular spaces as the brain's interstitial fluid drainage system. J Neurol Sci 398, 9-15 (2019).

24. Benveniste, H., Heerdt, P. M., Fontes, M., Rothman, D. L. \& Volkow, N. D. Glymphatic System Function in Relation to Anesthesia and Sleep States. Anesthesia and analgesia 128, 747-758 (2019).

25. Sachs, J. R., Zapadka, M. E. \& Elster, A. D. Orbital interstitial fluid: Evidence of a potential pathway for extracranial cerebrospinal fluid absorption. Journal of Computer Assisted Tomography 42, 497-501 (2018).

Page 12/14 
26. Joseph, C., Benhatzel, C., Hopper, O. \& Lockwood, M. Novel method utilizing arterial spin labeling single acquisition 3d echo planar imaging for determining CNS glymphatic clearance. Neurology 92 (2019).

27. Lim, M. M., Christy, A., Pollock, J. M., Case, B. \& Opel, R. A. Sleep disturbances correlate with perivascular spaces on brain MRI: A marker of impaired glymphatic clearance? Annals of Neurology 82, S209-S210 (2017).

28. Naganawa, S., Nakane, T., Kawai, H. \& Taoka, T. Gd-based Contrast Enhancement of the Perivascular Spaces in the Basal Ganglia. Magn Reson Med Sci 16, 61-65 (2017).

29. Naganawa, S., Nakane, T., Kawai, H. \& Taoka, T. Age Dependence of Gadolinium Leakage from the Cortical Veins into the Cerebrospinal Fluid Assessed with Whole Brain 3D-real Inversion Recovery MR Imaging. Magn Reson Med Sci 18, 163-169 (2019).

30. Ohashi, T., Naganawa, S., Ogawa, E., Katagiri, T. \& Kuno, K. Signal Intensity of the Cerebrospinal Fluid after Intravenous Administration of Gadoliniumbased Contrast Agents: Strong Contrast Enhancement around the Vein of Labbe. Magn Reson Med Sci 18, 194-199 (2019).

31. Rennels, M. L., Gregory, T. F., Blaumanis, O. R., Fujimoto, K. \& Grady, P. A. Evidence for a 'paravascular'fluid circulation in the mammalian central nervous system, provided by the rapid distribution of tracer protein throughout the brain from the subarachnoid space. Brain research 326, 47-63 (1985).

32. Rennels, M., Blaumanis, O. \& Grady, P. Rapid solute transport throughout the brain via paravascular fluid pathways. Advances in neurology $\mathbf{5 2}$, 431 (1990).

33. Eustace, S. \& Buff, B. Magnetic resonance imaging in drug-induced meningitis. Canadian Association of Radiologists journal= Journal l'Association canadienne des radiologistes 45, 463-465 (1994).

34. Kapoor, R., Liu, J., Devasenapathy, A. \& Gordin, V. Gadolinium encephalopathy after intrathecal gadolinium injection. Pain Physician 13, E321-E326 (2010).

35. Calvo, N. et al. Neurotoxicity from Intrathecal Gadolinium Administration (IT-Gad)(P3. 9-043) in Book Neurotoxicity from Intrathecal Gadolinium Administration (IT-Gad)(P3. 9-043) (ed.^(eds. Editor) (AAN Enterprises, 2019).

36. Arlt, S., Cepek, L., Rustenbeck, H. H., Prange, H. \& Reimers, C. D. Gadolinium encephalopathy due to accidental intrathecal administration of gadopentetate dimeglumine. Journal of neurology 254, 810 (2007).

37. Liberati, A. et al. The PRISMA statement for reporting systematic reviews and meta-analyses of studies that evaluate health care interventions: explanation and elaboration. Journal of clinical epidemiology 62, e1-e34 (2009).

38. Kim, S. Y. et al. Testing a tool for assessing the risk of bias for nonrandomized studies showed moderate reliability and promising validity. Journal of clinical epidemiology 66, 408-414 (2013).

\section{Figures}

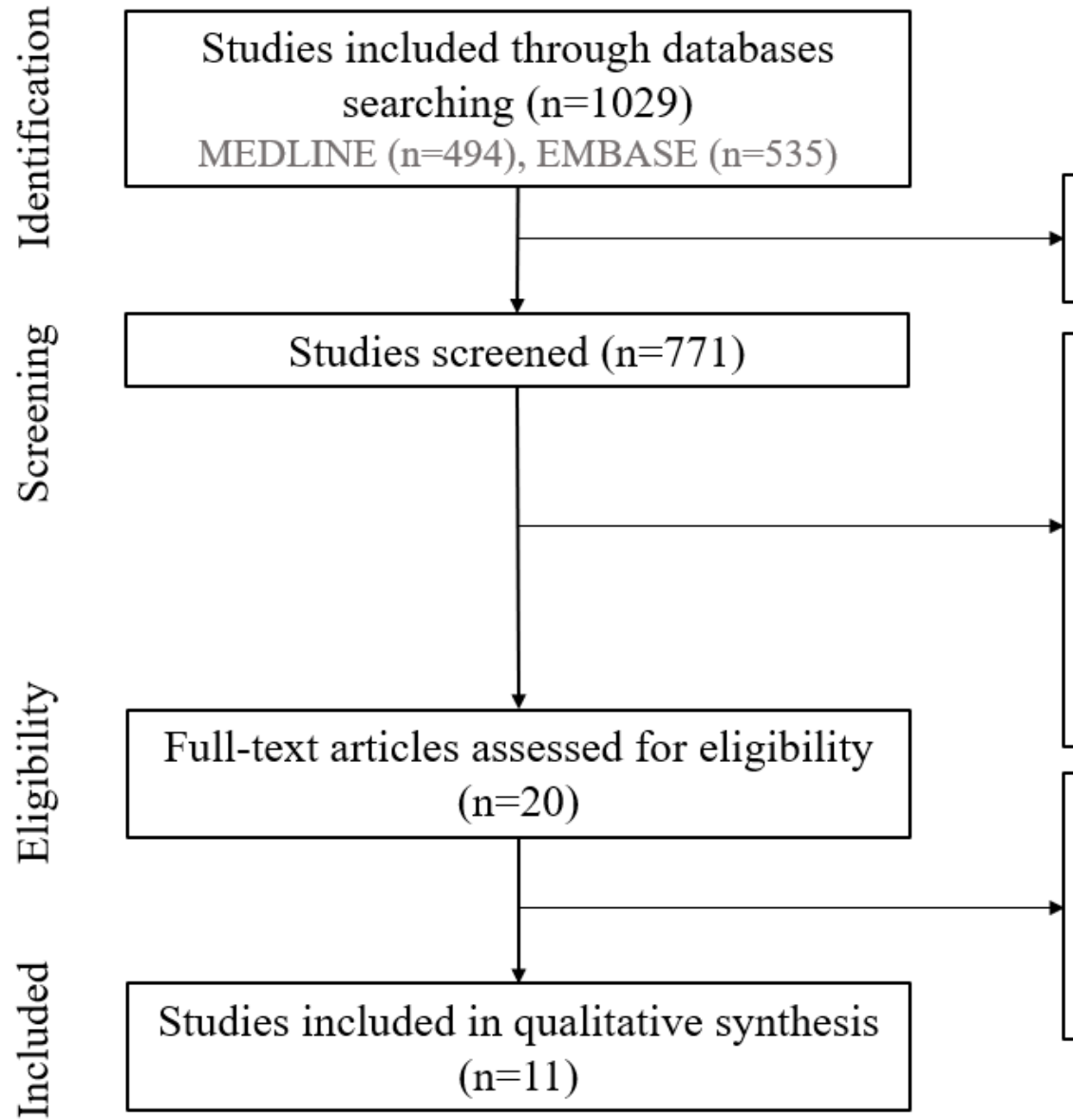
Duplicate articles removed $(\mathrm{n}=258)$

Excluded $(\mathrm{n}=751)$
Not in the field of interest $(\mathrm{n}=303)$
Review of articles $(\mathrm{n}=97)$
Case reports/series $(\mathrm{n}=209)$
Editorials/letters/abstracts $(\mathrm{n}=36)$
Contains Pathologic Patients $*(\mathrm{n}=63)$
Not human data $(\mathrm{n}=40)$
Did not perform MRI $(\mathrm{n}=3)$
Excluded $(\mathrm{n}=9)$
Not in the field of interest $(\mathrm{n}=2)$
Review of article $(\mathrm{n}=2)$
Editorials/letters/abstracts $(\mathrm{n}=2)$
Overlapping data $(\mathrm{n}=3)$

Page 13/14 
Figure 1

Flow diagram of the study selection process * The excluded patients encompassed degenerative cerebral disease, hydrocephalus, traumatic brain injury, cerebrovascular disease, cerebral demyelinating disease, cerebral metabolic disease, epilepsy, and/or solid brain tumor

\section{RISK OF BIAS}

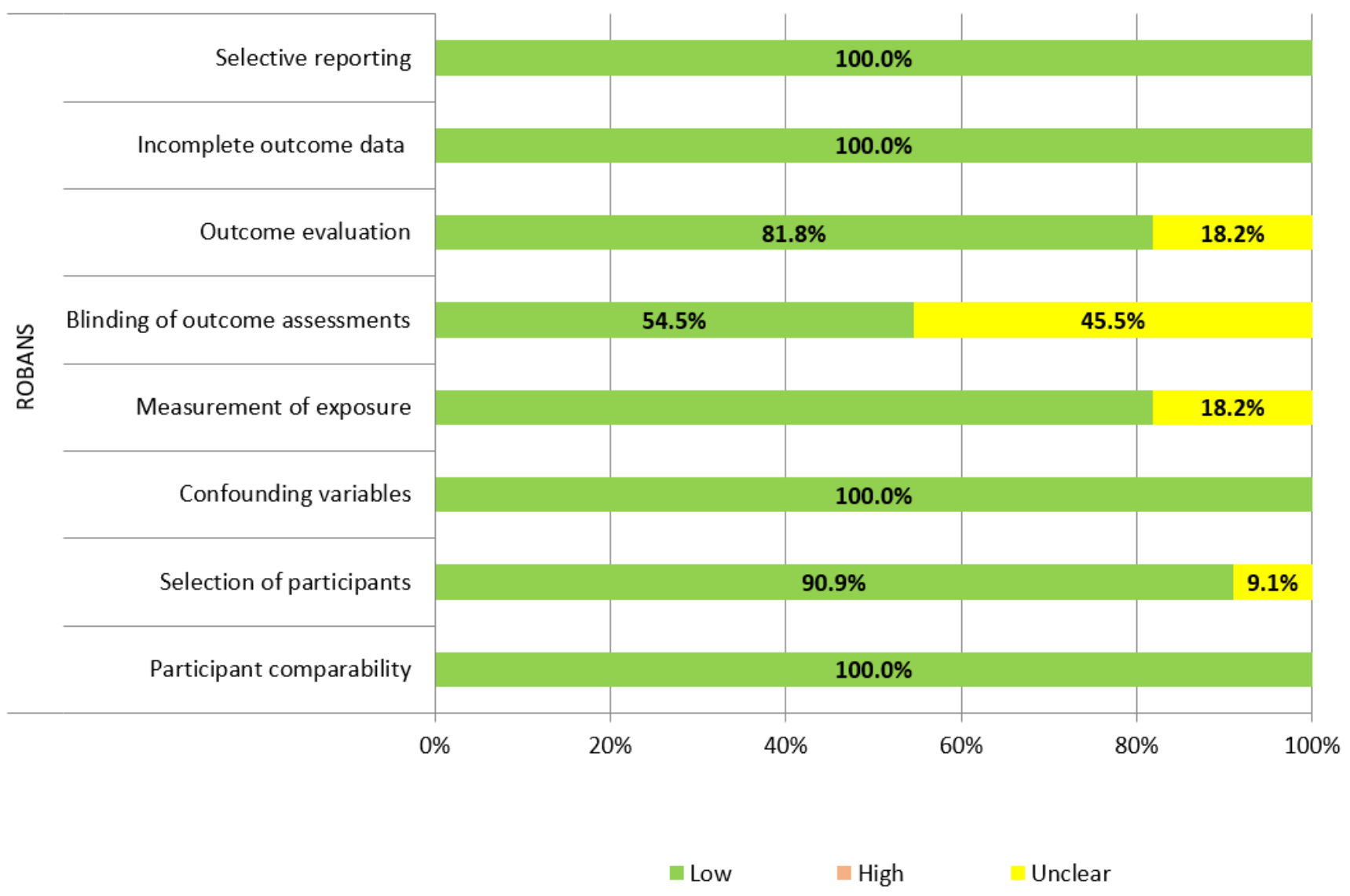

Figure 2

Risk of bias summary

\section{Supplementary Files}

This is a list of supplementary files associated with this preprint. Click to download.

- Supplementaryfigurelegend.docx

- supplementaryfigure1A.tif

- supplementaryfigure1B.tif 